\title{
TWO APPLICATIONS OF A BOUND ON THE HADAMARD PRODUCT WITH A CAUCHY MATRIX*
}

\author{
ROGER A. HORN ${ }^{\dagger}$ AND FUAD KITTANEH ${ }^{\ddagger}$
}

Dedicated to Hans Schneider on the occasion of his seventieth birthday

Abstract. A fundamental bound for a Hadamard multiplier operator of Cauchy type implies a known bound on the inverse of the operator $T: X \rightarrow A X-X B$ as well as a general perturbation bound involving the unitary factor in the polar decomposition; the latter bound leads to a perturbation bound on the positive semidefinite factor.

AMS(MOS) subject classification. 15A23, 15A45, 15A57, 15 A60.

Key words. Polar decomposition, unitary factor, positive semidefinite factor, unitarily invariant norm, Hadamard product, perturbation bound.

1. Introduction. Our main result (Theorem 3.2) is a general perturbation bound for the unitary factor in the polar decomposition of a nonsingular complex matrix. As in several other problems involving a unitarily invariant norm of a commutator-like expression, our result follows from a bound on a Hadamard multiplier operator of Cauchy type. One encounters a similar situation when seeking a bound on the norm of the solution operator for certain Sylvester equations (Theorem 2.1).

Let $M_{m, n}$ denote the set of $m$-by- $n$ complex matrices and set $M_{n} \equiv M_{n, n}$. We write the ordered singular values of any $A \in M_{m, n}$ as $\sigma_{\max }(A) \equiv \sigma_{1}(A) \geq \cdots \geq \sigma_{\min \{m, n\}}(A) \equiv$ $\sigma_{\min }(A) \geq 0$. We say that $A, B \in M_{m, n}$ are unitarily equivalent if there are unitary $V \in M_{m}$ and $W \in M_{n}$ such that $A=V B W$. A norm $\|\cdot\|$ on $M_{m, n}$ is unitarily invariant if $\|V A W\|=$ $\|A\|$ for all $A \in M_{m, n}$ and all unitary $V \in M_{m}, W \in M_{n}$; equivalently, such a norm is symmetric, which means that it always satisfies the bound $\|X A Y\| \leq \sigma_{1}(X)\|A\| \sigma_{1}(Y)$ [11, Problem 4, Section 3.5]. The Hadamard product of $A=\left[a_{i j}\right], B=\left[b_{i j}\right] \in M_{m, n}$ is $A \circ B \equiv\left[a_{i j} b_{i j}\right] \in M_{m, n}$. A Cauchy matrix has the special form $\left[\left(\alpha_{i}+\beta_{j}\right)^{-1}\right]$.

The unifying theme of this paper is Hadamard products with Cauchy matrices, for which the following fundamental and easily-proved bound is available.

THEOREM 1.1. Let $\left\{\alpha_{i}\right\}_{i=1}^{m},\left\{\beta_{i}\right\}_{i=1}^{n}$ be given complex numbers with positive real parts, and let $\|\cdot\|$ be a given norm on $M_{m, n}$. If $m \neq n$, assume that $\|\cdot\|$ is unitarily invariant; if $m=n$, assume either that $\|\cdot\|$ is unitarily invariant or that it is induced by an absolute norm on $\mathbb{C}^{n}$. Then

$$
\left\|\left[\frac{1}{\alpha_{i}+\beta_{j}}\right] \circ X\right\| \leq \frac{1}{\min \operatorname{Re} \alpha_{i}+\min \operatorname{Re} \beta_{j}}\|X\|
$$

for every $X \in M_{m, n}$.

* Received by the editors on 12 August 1997. Final manuscript accepted on 28 December 1997. Handling editor: Daniel Hershkowitz.

$\dagger$ Mathematics Department, University of Utah, Salt Lake City, Utah 84112, USA (rhorn@math.utah.edu).

$\ddagger$ Department of Mathematics, University of Jordan, Amman, Jordan. 
Proof. Set $D(t) \equiv \operatorname{diag}\left(e^{-\alpha_{1} t}, \ldots, e^{-\alpha_{m} t}\right)$ and $E(t) \equiv \operatorname{diag}\left(e^{-\beta_{1} t}, \ldots, e^{-\beta_{n} t}\right)$. Then

$$
\begin{aligned}
\left\|\left[\frac{1}{\alpha_{i}+\beta_{j}}\right] \circ X\right\| & =\left\|\left[\frac{x_{i j}}{\alpha_{i}+\beta_{j}}\right]\right\|=\left\|\left[\int_{0}^{\infty} e^{-\alpha_{i} t} x_{i j} e^{-\beta_{j} t} d t\right]\right\| \\
& =\left\|\int_{0}^{\infty} D(t) X E(t) d t\right\| \leq \int_{0}^{\infty}\|D(t) X E(t)\| d t \\
& \leq \int_{0}^{\infty} \sigma_{1}(D(t))\|X\| \sigma_{1}(E(t)) d t \\
& =\int_{0}^{\infty} e^{-\min \operatorname{Re} \alpha_{i} t}\|X\| e^{-\min \operatorname{Re} \beta_{j} t} d t \\
& =\int_{0}^{\infty} e^{-\left(\min \operatorname{Re} \alpha_{i}+\min \operatorname{Re} \beta_{j}\right) t}\|X\| d t \\
& =\frac{1}{\min \operatorname{Re} \alpha_{i}+\min \operatorname{Re} \beta_{j}}\|X\|
\end{aligned}
$$

The inequality in (2) holds for unitarily invariant norms because they are symmetric; if $m=n$ and the norm is induced by an absolute norm on $\mathbb{C}^{n}$, (2) holds because all such norms are submultiplicative and satisfy $\left\|\operatorname{diag}\left(\gamma_{1}, \ldots, \gamma_{n}\right)\right\|=\max \left|\gamma_{i}\right|$ on diagonal matrices [10, Theorem 5.6.37].

This approach to (1) is a specialization of [9, Theorem 5].

2. Application: A Bound for Solutions of $A X-B X=S$. If $A \in M_{m}$ and $B \in M_{n}$ have disjoint spectra, then $T_{A, B}(X) \equiv A X-X B=S$ has a unique solution $X \in M_{m, n}$ for every $S \in M_{m, n}$. Many authors have obtained bounds on the norm of the solution operator $T_{A, B}^{-1}: S \rightarrow X$ under various conditions on $A$ and $B$; for example, see [4], [5], and [6, Satz $5]$. We consider here a version of this problem that yields to a straightforward application of Theorem 1.1.

For a given nonnegative $\eta$, we say that two sets $S_{1}, S_{2} \subset \mathbb{C}$ are separated by a gap of width at least $\eta$ if there is a line $L$ in the complex plane such that $S_{1}$ lies on one side of $L$, $S_{2}$ lies on the other side of $L$, and the sum of the distances of $S_{1}$ and $S_{2}$ to $L$ is at least $\eta$.

THEOREM 2.1. Let $A \in M_{m}, B \in M_{n}$ be given normal matrices with respective spectra $\left\{\alpha_{i}\right\}_{i=1}^{m}$ and $\left\{\beta_{j}\right\}_{j=1}^{n}$, which we suppose to be separated by a gap of width at least $\eta>0$. Then $\|X\| \leq \eta^{-1}\|A X-X B\|$ for every unitarily invariant norm $\|\cdot\|$ on $M_{m, n}$.

Proof. Since the simultaneous transformations $A \rightarrow e^{i \theta} A+c I$ and $B \rightarrow e^{i \theta} B+c I$ preserve normality, the relevant geometry, and bounds for any real $\theta$ and any complex $c$, we may assume without loss of generality that $\operatorname{Re} \alpha_{i} \geq \eta / 2$ and $\operatorname{Re} \beta_{j} \leq-\eta / 2$ for all $i, j$. Write $A=V \mathcal{A} V^{*}$ and $B=W \mathcal{B} W^{*}$, where $V$ and $W$ are unitary, $\mathcal{A}=\operatorname{diag}\left(\alpha_{1}, \ldots, \alpha_{m}\right)$, and $\mathcal{B}=\operatorname{diag}\left(\beta_{1}, \ldots, \beta_{n}\right)$. Then

$$
\begin{aligned}
A X-X B & =V \mathcal{A} V^{*} X-X W \mathcal{B} W^{*}=V\{\mathcal{A} \underbrace{\left(V^{*} X W\right)}_{Y}-\underbrace{\left(V^{*} X W\right)}_{Y} \mathcal{B}\} W^{*} \\
& =V\{\mathcal{A} Y-Y \mathcal{B}\} W^{*}=V\left\{\left[\alpha_{i} y_{i j}-y_{i j} \beta_{j}\right]\right\} W^{*} \\
& =V\left\{\left[\alpha_{i}-\beta_{j}\right] \circ Y\right\} W^{*}
\end{aligned}
$$


and Theorem 1.1 gives

$$
\begin{aligned}
\|X\| & =\left\|V^{*} X W\right\|=\|Y\|=\left\|\left[\frac{1}{\alpha_{i}+\left(-\beta_{j}\right)}\right] \circ\left(\left[\alpha_{i}-\beta_{j}\right] \circ Y\right)\right\| \\
& \leq \frac{1}{\min \operatorname{Re} \alpha_{i}+\min \operatorname{Re}\left(-\beta_{j}\right)}\left\|\left[\alpha_{i}-\beta_{j}\right] \circ Y\right\| \\
& =\frac{1}{\min \operatorname{Re} \alpha_{i}-\max \operatorname{Re} \beta_{j}}\left\|V\left(\left[\alpha_{i}-\beta_{j}\right] \circ Y\right) W^{*}\right\| \\
& =\frac{1}{\min \operatorname{Re} \alpha_{i}-\max \operatorname{Re} \beta_{j}}\|A X-X B\| \leq \frac{1}{\eta}\|A X-X B\| .
\end{aligned}
$$

The bound in Theorem 2.1 is the same as that in [6, Satz 5] for the normal case, but our approach is quite different from that of Heinz.

3. Application: A General Perturbation Bound for the Unitary Factor. Every $A \in M_{n}$ has a singular value decomposition $A=V \Sigma W^{*}$, in which $V, W \in M_{n}$ are unitary and $\Sigma=\operatorname{diag}\left(\sigma_{1}(A), \ldots, \sigma_{n}(A)\right)$. Writing $A=\left(V W^{*}\right)\left(W \Sigma W^{*}\right)$ gives a polar decomposition $A=U(A)|A|$ in which $U(A)=V W^{*}$ is unitary and $|A|=W \Sigma W^{*}$ is positive semidefinite. Since $A^{*}=W \sum V^{*}=\left(W V^{*}\right)\left(V \Sigma V^{*}\right)=U(A)^{*}\left(V \Sigma V^{*}\right)$, we see that $A^{*}$ always has a polar decomposition in which the unitary factor is $U(A)^{*}$. If $Y, Z \in M_{n}$ are unitary, then

$$
Y A Z^{*}=\left(Y U(A) Z^{*}\right)\left(Z|A| Z^{*}\right)
$$

gives a polar decomposition of any unitary equivalence of a matrix that has a given polar decomposition.

The positive semidefinite factor in the polar decomposition of $A \in M_{n}$ is always uniquely determined as $|A|=\left(A^{*} A\right)^{1 / 2}$, but if $A$ is singular there are many choices for the unitary factor. If $A$ is nonsingular, however, then so is $|A|$ and the unitary factor $U(A)=A|A|^{-1}$ is uniquely determined.

Let $\tilde{A}, \tilde{B} \in M_{2 n}$ be Hermitian and nonsingular, and have respective spectra $\left\{ \pm \alpha_{i}\right\}_{i=1}^{n}$ and $\left\{ \pm \beta_{j}\right\}_{j=1}^{n}$ with positive $\alpha_{i}, \beta_{j}$. Let $I_{n}$ denote the $n$-by- $n$ identity matrix. Write $\tilde{A}=$ $V \mathcal{A} V^{*}$ and $\tilde{B}=W \mathcal{B} W^{*}$, where $V, W \in M_{2 n}$ are unitary, $\mathcal{A}=\operatorname{diag}\left(\alpha_{1}, \ldots, \alpha_{n},-\alpha_{1}, \ldots,-\alpha_{n}\right)$, and $\mathcal{B}=\operatorname{diag}\left(\beta_{1}, \ldots, \beta_{n},-\beta_{1}, \ldots,-\beta_{n}\right)$. Since $U(\mathcal{A})=U(\mathcal{B})=I_{n} \oplus\left(-I_{n}\right) \equiv \mathcal{I}$, we have $U(\tilde{A})=V \mathcal{I} V^{*}$ and $U(\tilde{B})=W \mathcal{I} W^{*}$. For any $Y \in M_{2 n}$, set

$$
X \equiv V^{*} Y W=\left[\begin{array}{ll}
X_{11} & X_{12} \\
X_{21} & X_{22}
\end{array}\right], \quad X_{i j} \in M_{n}
$$

and compute

$$
\begin{aligned}
\tilde{A} Y-Y \tilde{B} & =V\left\{\mathcal{A}\left(V^{*} Y W\right)-\left(V^{*} Y W\right) \mathcal{B}\right\} W^{*} \\
& =V\{\mathcal{A} X-X \mathcal{B}\} W^{*} \\
& =V\left[\begin{array}{lr}
{\left[\alpha_{i}-\beta_{j}\right] \circ X_{11}} & {\left[\alpha_{i}+\beta_{j}\right] \circ X_{12}} \\
{\left[\alpha_{i}+\beta_{j}\right] \circ\left(-X_{21}\right)} & -\left[\alpha_{i}-\beta_{j}\right] \circ X_{22}
\end{array}\right] W^{*} .
\end{aligned}
$$


For any $Z \in M_{2 n}$, write

$$
Z=\left[\begin{array}{ll}
Z_{11} & Z_{12} \\
Z_{21} & Z_{22}
\end{array}\right], \quad Z_{i j} \in M_{n}
$$

let

$$
\hat{Z} \equiv\left[\begin{array}{ll}
0 & Z_{12} \\
Z_{21} & 0
\end{array}\right]
$$

denote the pinched matrix obtained from the block matrix $Z$, and observe that pinching is unitarily invariant norm decreasing:

$$
\|\hat{Z}\|=\left\|\frac{1}{2}(Z-\mathcal{I} Z \mathcal{I})\right\| \leq \frac{1}{2}(\|Z\|+\|\mathcal{I} Z \mathcal{I}\|)=\frac{1}{2}(\|Z\|+\|Z\|)=\|Z\|
$$

for any unitarily invariant norm $\|\cdot\|$. Finally, compute

$$
\begin{aligned}
& \|U(\tilde{A}) Y-Y U(\tilde{B})\|=\left\|\left(V \mathcal{I} V^{*}\right)\left(V X W^{*}\right)-\left(V X W^{*}\right)\left(W \mathcal{I} W^{*}\right)\right\| \\
& =\|\mathcal{I} X-X \mathcal{I}\|=\left\|\left[\begin{array}{ll}
0 & 2 X_{12} \\
-2 X_{21} & 0
\end{array}\right]\right\| \\
& =2\left\|\begin{array}{ll}
0 & {\left[\frac{\alpha_{i}+\beta_{j}}{\alpha_{i}+\beta_{j}}\right] \circ X_{12}} \\
{\left[\frac{\alpha_{i}+\beta_{j}}{\alpha_{i}+\beta_{j}}\right] \circ\left(-X_{21}\right)} & 0
\end{array}\right\| \\
& \leq \frac{2}{\min \alpha_{i}+\min \beta_{j}}\left\|\left[\begin{array}{cc}
0 & {\left[\alpha_{i}+\beta_{j}\right] \circ X_{12}} \\
-\left[\alpha_{i}+\beta_{j}\right] \circ X_{21} & 0
\end{array}\right]\right\| \\
& \leq \frac{2}{\min \alpha_{i}+\min \beta_{j}}\left\|\left[\begin{array}{lr}
{\left[\alpha_{i}-\beta_{j}\right] \circ X_{11}} & {\left[\alpha_{i}+\beta_{j}\right] \circ X_{12}} \\
{\left[\alpha_{i}+\beta_{j}\right] \circ\left(-X_{21}\right)} & -\left[\alpha_{i}-\beta_{j}\right] \circ X_{11}
\end{array}\right]\right\| \\
& =\frac{2}{\min \alpha_{i}+\min \beta_{j}}\|\tilde{A} Y-Y \tilde{B}\| \text {. }
\end{aligned}
$$

The inequalities in (3) and (4) are ensured by Theorem 1.1 and pinching, respectively.

We have proved the following result involving unitarily invariant norms and the unitary factors of certain structured Hermitian matrices.

LEMMA 3.1. Suppose Hermitian matrices $\tilde{A}, \tilde{B} \in M_{2 n}$ have respective spectra $\left\{ \pm \alpha_{i}\right\}_{i=1}^{n}$ and $\left\{ \pm \beta_{i}\right\}_{i=1}^{n}$ with positive $\alpha_{i}, \beta_{i}$. Then

$$
\|U(\tilde{A}) Y-Y U(\tilde{B})\| \leq \frac{2}{\min \alpha_{i}+\min \beta_{j}}\|\tilde{A} Y-Y \tilde{B}\|
$$

for all $Y \in M_{2 n}$ and every unitarily invariant norm $\|\cdot\|$.

Hermitian matrices with the structure assumed in Lemma 3.1 arise naturally in problems involving singular values and unitarily invariant norms. Consider nonsingular $A, B \in$ $M_{n}$ and the $2 n$-by- $2 n$ Hermitian matrices

$$
\tilde{A} \equiv\left[\begin{array}{ll}
0 & A \\
A^{*} & 0
\end{array}\right] \text { and } \tilde{B} \equiv\left[\begin{array}{ll}
0 & B \\
B^{*} & 0
\end{array}\right]
$$


which have respective spectra $\left\{ \pm \sigma_{i}(A)\right\}_{i=1}^{n}$ and $\left\{ \pm \sigma_{i}(B)\right\}_{i=1}^{n}$ [10, Theorem 7.3.7]. Block matrices of this form were used by Jordan in one of the earliest approaches to the singular value decomposition [12]. The polar decomposition of such matrices is easily computed: $\tilde{A}=U(\tilde{A})|\tilde{A}|$, with

$$
U(\tilde{A})=\left[\begin{array}{ll}
0 & U(A) \\
U(A)^{*} & 0
\end{array}\right] \text { and }|\tilde{A}|=\left[\begin{array}{ll}
\left|A^{*}\right| & 0 \\
0 & |A|
\end{array}\right]
$$

For $X \in M_{n}$, set $Y \equiv\left[\begin{array}{ll}X & 0 \\ 0 & X\end{array}\right]$ and compute

$$
\begin{aligned}
\tilde{A} Y & -Y \tilde{B}=\left[\begin{array}{ll}
0 & A X-X B \\
A^{*} X-X B^{*} & 0
\end{array}\right] \\
& =\left[\begin{array}{ll}
A X-X B & 0 \\
0 & A^{*} X-X B^{*}
\end{array}\right]\left[\begin{array}{ll}
0 & I \\
I & 0
\end{array}\right]
\end{aligned}
$$

and

$$
\begin{aligned}
U(\tilde{A}) Y & -Y U(\tilde{B})=\left[\begin{array}{ll}
0 & U(A) X-X U(B) \\
U(A)^{*} X-X U(B)^{*} & 0
\end{array}\right] \\
& =\left[\begin{array}{ll}
U(A) X-X U(B) & 0 \\
0 & U(A)^{*} X-X U(B)^{*}
\end{array}\right]\left[\begin{array}{ll}
0 & I \\
I & 0
\end{array}\right]
\end{aligned}
$$

Finally, observe that

$$
U(A)^{*} X-X U(B)^{*}=-U(A)^{*}\{U(A) X-X U(B)\} U(B)^{*}
$$

so the singular values of $U(A) X-X U(B)$ and $U(A)^{*} X-X U(B)^{*}$ are the same. However, $A X-X B$ and $A^{*} X-X B^{*}$ need not have the same singular values. We now have all the elements required to state and prove a general perturbation theorem for the unitary factor in the polar decomposition of a nonsingular complex matrix.

THEOREM 3.2. Let nonsingular $A, B \in M_{n}$ be given. Then

$$
\begin{aligned}
& \|(U(A) X-X U(B)) \oplus(U(A) X-X U(B))\| \\
& \leq \frac{2}{\sigma_{\min }(A)+\sigma_{\min }(B)}\left\|(A X-X B) \oplus\left(A^{*} X-X B^{*}\right)\right\|
\end{aligned}
$$

for all $X \in M_{n}$ and every unitarily invariant norm $\|\cdot\|$.

Proof. Use (5) and (6) in Lemma 3.1.

The bound in (7) is sharp, as the inequality becomes an equality when both $A$ and $B$ are unitary.

For the spectral norm $\|X\| \|_{2} \equiv \sigma_{\max }(X)$ and the Schatten $p$-norms $\|X\|_{p} \equiv\left(\sum_{i=1}^{n} \sigma_{i}(X)^{p}\right)^{1 / p}$, $p \geq 1$, both sides of the inequality $(7)$ are explicit functions of the singular values. 
Corollary 3.3. Let nonsingular $A, B \in M_{n}$ be given. Then

$$
\leq \frac{\|U(A) X-X U(B)\|_{2}}{\leq} \frac{2}{\sigma_{\min }(A)+\sigma_{\min }(B)} \max \left\{\|\mid A X-X B\|\left\|_{2},\right\| A^{*} X-X B^{*} \|_{2}\right\}
$$

and

$$
\begin{aligned}
& \|U(A) X-X U(B)\|_{p} \\
\leq & \frac{2^{1-\frac{1}{p}}}{\sigma_{\min }(A)+\sigma_{\min }(B)}\left(\|A X-X B\|_{p}^{p}+\left\|A^{*} X-X B^{*}\right\|_{p}^{p}\right)^{1 / p}
\end{aligned}
$$

for all $X \in M_{n}$ and any $p \geq 1$.

If $A^{*} X-X B^{*}$ and $A X-X B$ are unitarily equivalent, then (7), (8), and (9) can be simplified.

Corollary 3.4. Let $A, B, X \in M_{n}$ be given, suppose $A$ and $B$ are nonsingular, and suppose $A^{*} X-X B^{*}$ and $A X-X B$ are unitarily equivalent. Then

$$
\|U(A) X-X U(B)\| \leq \frac{2}{\sigma_{\min }(A)+\sigma_{\min }(B)}\|A X-X B\|
$$

for every unitarily invariant norm $\|\cdot\|$. In particular, (10) holds if $X$ is Hermitian or skewHermitian and commutes with both $A$ and $B$.

Proof. Consider (7) for a Ky Fan $k$-norm, which is the sum of the $k$ largest singular values. If we take $k=2 m$, then ( 7 ) gives

$$
\begin{aligned}
\sum_{i=1}^{m} \sigma_{i}(U(A) X & -X U(B)) \\
& =\frac{1}{2} \sum_{i=1}^{2 m} \sigma_{i}((U(A) X-X U(B)) \oplus(U(A) X-X U(B))) \\
& \leq \frac{1}{\sigma_{\min }(A)+\sigma_{\min }(B)} \sum_{i=1}^{2 m} \sigma_{i}\left((A X-X B) \oplus\left(A^{*} X-X B^{*}\right)\right) \\
& =\frac{2}{\sigma_{\min }(A)+\sigma_{\min }(B)} \sum_{i=1}^{m} \sigma_{i}(A X-X B)
\end{aligned}
$$

for $m=1, \ldots, n$. The Ky Fan Dominance Theorem [11, Corollary 3.5.9] now ensures that

$$
\|U(A) X-X U(B)\| \leq \frac{2}{\sigma_{\min }(A)+\sigma_{\min }(B)}\|A X-X B\|
$$

for every unitarily invariant norm $\|\cdot\|$. The final assertion follows from observing that $A^{*} X-$ $X B^{*}= \pm(A X-X B)^{*}$ under the stated conditions.

If we take $X=I$ in $(10)$, we obtain a bound

$$
\|U(A)-U(B)\| \leq \frac{2}{\sigma_{\min }(A)+\sigma_{\min }(B)}\|A-B\|
$$


proved by R.-C. Li [13], who derived it from the Hermitian case of Theorem 2.1. Other bounds on $\|U(A)-U(B)\|$ are known; see [2], [7], [14] and references therein. However, most of these bounds are either asymptotic or valid only under restrictions; they are based on knowledge of the derivative of the map $A \rightarrow U(A)$.

One final special case of $(7)$ is worth noting for the Schatten $p$-norms. The Frobenius norm $\|\cdot\|_{2}$ is simultaneously the Schatten 2-norm and the sum of the squares of the moduli of the entries of its argument. For any $A, B, X \in M_{n}$ we have

$$
\begin{aligned}
\|A X-X B\|_{2}^{2} & =\operatorname{tr}(A X-X B)^{*}(A X-X B) \\
& =\operatorname{tr}\left(X^{*} A^{*} A X+X B B^{*} X^{*}\right)-\operatorname{tr}\left(A^{*} X B X^{*}+A X B^{*} X^{*}\right),
\end{aligned}
$$

in which the second term in (12) is invariant under the substitutions $A \rightarrow A^{*}$ and $B \rightarrow B^{*}$; the first term has the same invariance if $A$ and $B$ are normal. Thus, for normal $A$ and $B$, the Frobenius norms of $A X-X B$ and $A^{*} X-X B^{*}$ are the same. For any $p \in(1, \infty)$ and for normal $A$ and $B$ it follows from Theorem 2.7 of [1] that $\left\|A^{*} X-X B^{*}\right\|_{p} \leq c_{p}\|A X-X B\|$, where $c_{p}$ is a universal constant that depends only on $p$; we have just observed that one may take $c_{2}=1$. The bounds in Corollary 3.3 yield the following corollary.

Corollary 3.5. Let $A, B, X \in M_{n}$ be given with $A$ and $B$ nonsingular and normal. Then

$$
\|U(A) X-X U(B)\|_{2} \leq \frac{2}{\sigma_{\min }(A)+\sigma_{\min }(B)}\|A X-X B\|_{2}
$$

and for each $p \in(1, \infty)$ there is a constant $c_{p}$ depending only on $p$ such that

$$
\|U(A) X-X U(B)\|_{p} \leq \frac{2^{1-\frac{1}{p}}\left(1+c_{p}^{p}\right)^{\frac{1}{p}}}{\sigma_{\min }(A)+\sigma_{\min }(B)}\|A X-X B\|_{p}
$$

Our Theorem 3.2 is a generalization of R.-C. Li's bound (11) in the spirit of an ongoing program to strengthen a variety of norm inequalities that is described eloquently in [3]; its authors opine that "the insertion of $X$ is no idle generalization".

4. Perturbation of the Positive Semidefinite Factor. The problem of bounding ||$|A|-|B|||$ is a natural companion to the problem studied in the preceding section, and our results give some interesting new bounds. A straightforward computation shows that

$$
\begin{aligned}
|A|-|B| & =\frac{1}{2}(U(A)+U(B))^{*}(A-B)+\frac{1}{2}(U(A)-U(B))^{*}(A+B) \\
& =U(A)^{*}(A-B)+(U(A)-U(B))^{*} B \\
& =U(B)^{*}(A-B)+(U(A)-U(B))^{*} A
\end{aligned}
$$

for all nonsingular $A, B \in M_{n}$. Since every unitarily invariant norm is symmetric, we have

$$
\||A|-|B|\| \leq\left\{\begin{array}{l}
\|A-B\|+\frac{1}{2} \sigma_{\max }(A+B)\|U(A)-U(B)\| \\
\|A-B\|+\sigma_{\max }(B)\|U(A)-U(B)\| \\
\|A-B\|+\sigma_{\max }(A)\|U(A)-U(B)\|
\end{array}\right.
$$


Continuity and the bound in (11) then give our last result.

THEOREM 4.1. Let $A, B \in M_{n}$ be given, with at least one of $A$ and $B$ nonsingular. Then

$$
\begin{aligned}
& \||A|-|B|\| \\
\leq & \left(1+\frac{\min \left\{\sigma_{\max }(A+B), 2 \sigma_{\max }(A), 2 \sigma_{\max }(B)\right\}}{\sigma_{\min }(A)+\sigma_{\min }(B)}\right)\|A-B\|
\end{aligned}
$$

for every unitarily invariant norm $\|\cdot\|$ on $M_{n}$.

Related inequalities involving $|A| X-X|B|, A X-X B$, and $A^{*} X-X B^{*}$ can be derived in a similar way by using the identities

$$
\begin{aligned}
|A| X-X|B| & =U(A)^{*}(A X-X B)+\left(U(A)^{*} X-X U(B)^{*}\right) B \\
& =\left(A^{*} X-X B^{*}\right) U(B)+A^{*}(U(A) X-X U(B))
\end{aligned}
$$

the resulting bounds

$$
\||A| X-X|B|\| \leq\left\{\begin{array}{l}
\|A X-X B\|+\sigma_{\max }(B)\|U(A) X-X U(B)\| \\
\left\|A^{*} X-X B^{*}\right\|+\sigma_{\max }(A)\|U(A) X-X U(B)\|,
\end{array}\right.
$$

and Theorem 3.2 and its corollaries.

The coefficient of $\|A-B\|$ in (14) is independent of $n$, but it depends on $A$ and $B$. For a survey of bounds in which that coefficient is independent of $A$ and $B$ but may depend on $n$ (that is, bounds of the form $\||A|-|B|\| \leq c_{n}\|A-B\|$ ), see [2, pp. 270-274] and its references, as well as [1] and [8]. For example, for the Frobenius norm one may take $c_{n}=\sqrt{2}$, but for the spectral norm it is necessary that $c_{n} \geq \frac{1}{2} \sqrt{\log _{2} n}$.

\section{REFERENCES}

[1] A. Abdessemed and E.B. Davies, Some commutator estimates in the Schatten classes, J. London Math. Soc., 39:299-308, 1989.

[2] R. Bhatia, Matrix factorisations and their perturbations, Linear Algebra Appl., 197/198:245-276, 1994.

[3] R. Bhatia and C. Davis, A Cauchy-Schwarz Inequality for Operators with Applications, Linear Algebra Appl., 223/224:119-129, 1995.

[4] R. Bhatia, C. Davis, and A. McIntosh, Perturbations of Spectral Subspaces and Solution of Linear Operator Equations, Linear Algebra Appl.,52/53:45-67, 1983.

[5] C. Davis and W.M. Kahan, The rotation of eigenvectors by a perturbation. III, SIAM J. Numer. Anal., 7:1-46, 1970.

[6] E. Heinz, Beiträge zur Störungstheorie der Spektralzerlegung, Math. Annalen, 123:415438, 1951.

[7] N.J. Higham, The matrix sign decomposition and its relation to the polar decomposition, Linear Algebra Appl., 212/213:3-20, 1994.

[8] F. Kittaneh, On the continuity of the absolute value map in the Schatten classes, Linear Algebra Appl., 118:61-68, 1989.

[9] R.A. Horn, Norm bounds for Hadamard products and an arithmetic-geometric mean inequality for unitarily invariant norms, Linear Algebra Appl., 223/224:355-361, 1995. 


\section{ELA}

[10] R.A. Horn and C.R. Johnson, Matrix Analysis, Cambridge University Press, New York, 1985.

[11] R.A. Horn and C.R. Johnson, Topics in Matrix Analysis, Cambridge University Press, New York, 1991.

[12] C. Jordan, Mémoire sur les Formes Bilinéaires, J. Math. Pures Appl. (Ser. 2), 19:35-54, 1874.

[13] R.-C. Li, New perturbation bounds for the unitary polar factor, SIAM J. Matrix Anal. Appl., 16:327-332, 1995.

[14] R. Mathias, Perturbation bounds for the polar decomposition, SIAM J. Matrix Anal. Appl., 14:588-597, 1993. 